\title{
Sharp results concerning disjoint cross-intersecting families
}

\author{
Peter Frankl;, Andrey Kupavskii ${ }^{\dagger}$
}

\begin{abstract}
For an $n$-element set $X$ let $\left(\begin{array}{c}X \\ k\end{array}\right)$ be the collection of all its $k$-subsets. Two families of sets $\mathcal{A}$ and $\mathcal{B}$ are called cross-intersecting if $A \cap B \neq \emptyset$ holds for all $A \in \mathcal{A}, B \in \mathcal{B}$. Let $f(n, k)$ denote the maximum of $\min \{|\mathcal{A}|,|\mathcal{B}|\}$ where the maximum is taken over all pairs of disjoint, cross-intersecting families $\mathcal{A}, \mathcal{B} \subset\left(\begin{array}{c}{[n]} \\ k\end{array}\right)$. Let $c=\log _{2} e$. We prove that $f(n, k)=\left\lfloor\frac{1}{2}\left(\begin{array}{l}n-1 \\ k-1\end{array}\right)\right\rfloor$ essentially iff $n>c k^{2}$ (cf. Theorem 1.4 for the exact statement). Let $f^{*}(n, k)$ denote the same maximum under the additional restriction that the intersection of all members of both $\mathcal{A}$ and $\mathcal{B}$ are empty. For $k \geq 5$ and $n \geq k^{3}$ we show that $f^{*}(n, k)=$ $\left\lfloor\frac{1}{2}\left(\left(\begin{array}{c}n-1 \\ k-1\end{array}\right)-\left(\begin{array}{c}n-2 k \\ k-1\end{array}\right)\right)\right\rfloor+1$ and the restriction on $n$ is essentially sharp (cf. Theorem 5.4).
\end{abstract}

\section{Introduction}

Let $[n]=\{1,2,3, \ldots, n\}$ be the standard $n$-element set and let $2^{[n]}$ denote its power set. A subset $\mathcal{F} \subset 2^{[n]}$ is called a family. For $0 \leq k \leq n$ let $\left(\begin{array}{c}{[n]} \\ k\end{array}\right)=\{F \subset[n]:|F|=k\}$. Subsets of $\left(\begin{array}{c}{[n]} \\ k\end{array}\right)$ are called $k$-uniform. A family $\mathcal{F}$ is called intersecting if $F \cap F^{\prime} \neq \emptyset$ for all $F, F^{\prime} \in \mathcal{F}$. Let us state one of the central results in extremal set theory

Erdös-Ko-Rado Theorem $([\mathbf{E K R}])$. Suppose that $\mathcal{F} \subset 2^{[n]}$ is intersecting. Then (i) and (ii) hold.

\footnotetext{
*Rényi Institute, Budapest, Hungary

${ }^{\dagger}$ University of Oxford and Moscow Institute of Physics and Technology; Email: kupavskii@yandex.ru The research was supported by the Advanced Postdoc.Mobility grant no. P300P2_177839 of the Swiss National Science Foundation.
} 
(i) $|\mathcal{F}| \leq 2^{n-1}$.

(ii) Assuming that $\mathcal{F}$ is $k$-uniform and $n \geq 2 k$ one has

$$
|\mathcal{F}| \leq\left(\begin{array}{l}
n-1 \\
k-1
\end{array}\right)
$$

We should mention that (i) is a trivial consequence of the fact that $F \in \mathcal{F}$ implies $([n] \backslash F) \notin \mathcal{F}$. There are many different proofs for (1.1). E.g., [D], [Ka2], [Py], [FF1], [HK], to mention a few.

Definition 1.1. If for some $x \in[n], x \in F$ for all $F \in \mathcal{F}$ then $\mathcal{F}$ is called a star.

The full star $\mathcal{S}_{x}=\left\{S \in\left(\begin{array}{c}{[n]} \\ k\end{array}\right): x \in S\right\}$ shows that (1.1) is sharp.

Hilton-Milner Theorem ([HM] $)$. Suppose that $n>2 k, \mathcal{F} \subset\left(\begin{array}{c}{[n]} \\ k\end{array}\right)$ is intersecting and $\mathcal{F}$ is not a star. Then

$$
|\mathcal{F}| \leq\left(\begin{array}{l}
n-1 \\
k-1
\end{array}\right)-\left(\begin{array}{c}
n-k-1 \\
k-1
\end{array}\right)+1
$$

There are many known proofs for this important result as well. E.g. [FF2, [F87, [KZ], to mention a few.

Trying to prove results about intersecting families one arrives naturally at the following notion.

Definition 1.2. Two families $\mathcal{A}, \mathcal{B}$ are called cross-intersecting if $A \cap B \neq \emptyset$ for all $A \in \mathcal{A}, B \in \mathcal{B}$.

Noting that for $\mathcal{A}=\mathcal{B}$ the cross-intersecting property reduces to $\mathcal{A}$ being intersecting, one can generalize (i) to:

$$
|\mathcal{A}|+|\mathcal{B}| \leq 2^{n}
$$

whenever $\mathcal{A}, \mathcal{B} \subset 2^{[n]}$ are cross-intersecting. Although the bound $|\mathcal{A}|+|\mathcal{B}| \leq$ $\left(\begin{array}{l}n \\ k\end{array}\right)$ is true, for $n>2 k$ it is not sufficient to derive (1.1). However, considering products does the job. 
Pyber Theorem [Py]. Suppose that $\mathcal{A}, \mathcal{B} \subset\left(\begin{array}{c}{[n]} \\ k\end{array}\right)$ are cross-intersecting, $n \geq 2 k$. Then

$$
|\mathcal{A}| \cdot|\mathcal{B}| \leq\left(\begin{array}{l}
n-1 \\
k-1
\end{array}\right)^{2}
$$

Let us mention [FK1] where a short proof of (1.1) is given.

The following natural question was first considered in $[\mathrm{DF}]$.

Determine or estimate $f(n) \stackrel{\text { def }}{=} \min \{|\mathcal{A}|,|\mathcal{B}|\}$ where $\mathcal{A}, \mathcal{B} \subset 2^{[n]}$ are crossintersecting and $\mathcal{A} \cap \mathcal{B}=\emptyset$. The following, rather surprising result was proved in $[\mathrm{DF}]$.

$$
f(n)<\frac{3-\sqrt{5}}{2} \cdot 2^{n}
$$

and the constant $\frac{3-\sqrt{5}}{2}$ is optimal.

The second author was the first to consider the corresponding function $f(n, k)$ for $k$-uniform families.

Conjecture 1.3 ([K17]). Suppose that $n>2 k \geq 4$. Let $f(n, k)=$ $\max \min \{|\mathcal{A}|,|\mathcal{B}|\}$ where the maximum is over all disjoint and cross-intersecting families $\mathcal{A}, \mathcal{B} \subset\left(\begin{array}{c}{[n]} \\ k\end{array}\right)$. Then

$$
f(n, k)=\left\lfloor\frac{1}{2}\left(\begin{array}{l}
n-1 \\
k-1
\end{array}\right)\right\rfloor .
$$

A few months later Huang and independently the present authors disproved (1.5) for $n<k^{2}$ (cf. [H]). On the positive side Huang [H] proved (1.5) for $n>2 k^{2}$.

In the present paper we determine the range where (1.5) holds almost completely. Set $c=\log _{2} e$ and note that $c<3 / 2$.

Theorem 1.4. (i) If $n \geq c k^{2}+(2-c) k$ then (1.5) is true.

(ii) If $n \leq c k^{2}-2 c k+1$ then (1.5) fails.

\section{Tools of proofs}

For a family $\mathcal{F}$ and a positive integer $l$ define the $l$-shadow $\sigma^{(l)}(\mathcal{F})=\{G$ : $|G|=l, \exists F \in \mathcal{F}, G \subset F\}$. In other words, $\sigma^{(l)}(\mathcal{F})=\bigcup_{F \in \mathcal{F}}\left(\begin{array}{c}F \\ l\end{array}\right)$. 
One of the most important results in extremal set theory is the KruskalKatona Theorem. For given positive integers $m, k, l, k>l$ it determines the minimum of $\left|\sigma^{(l)}(\mathcal{F})\right|$ where $\mathcal{F}$ is $k$-uniform and $|\mathcal{F}|=m$.

It was Daykin's proof [D] of the Erdös-Ko-Rado Theorem that established the connection between these two important theorems. To state this connection in the more general setting of pairs of cross-intersecting families let us define the lexicographic order, $<_{L}$ on $\left(\begin{array}{c}{[n]} \\ t\end{array}\right), 1 \leq t \leq n$. For $G, H \in\left(\begin{array}{c}{[n]} \\ t\end{array}\right)$,

$$
G<_{L} H \text { iff } \min \{i: i \in G \backslash H\}<\min \{j: j \in H \backslash G\} .
$$

That is, $\{1,2,77\}<{ }_{L}\{1,3,4\}$.

For fixed $t$ and $m, 1 \leq m<\left(\begin{array}{l}n \\ t\end{array}\right)$ let $\mathcal{L}(n, t, m)$ denote the initial segment, the first $m$ subsets of size $t$ in the lexicographic order.

Let now $a, b$ be positive integers, $a+b<n$. Hilton [Hi] observed that $\mathcal{A} \subset\left(\begin{array}{c}{[n]} \\ a\end{array}\right)$ and $\mathcal{B} \subset\left(\begin{array}{c}{[n]} \\ b\end{array}\right)$ are cross-intersecting iff

$$
\mathcal{A} \cap \sigma^{(a)}\left(\mathcal{B}^{c}\right)=\emptyset
$$

where $\mathcal{B}^{c}=\{[n] \backslash B: B \in \mathcal{B}\}$ is the family of complements. This permits the following equivalent formulation of the Kruskal-Katona Theorem.

Kruskal-Katona Theorem ([Ka1,$[\overline{\mathbf{K r}}])$. Let $a, b$ be positive integers, $n>a+b$. Suppose that $\mathcal{A} \subset\left(\begin{array}{c}{[n]} \\ a\end{array}\right)$ and $\mathcal{B} \subset\left(\begin{array}{c}{[n]} \\ b\end{array}\right)$ are cross-intersecting. Then $\mathcal{L}(n, a,|\mathcal{A}|)$ and $\mathcal{L}(n, b,|\mathcal{B}|)$ are cross-intersecting too.

To get the reader familiar with this formulation let us show an easy consequence that we need later.

Corollary 2.1. Let $n>2 k>0$ and suppose that $\mathcal{G} \subset\left(\begin{array}{c}{[n-1]} \\ k-1\end{array}\right)$ and $\mathcal{H} \subset\left(\begin{array}{c}{[n-1]} \\ k\end{array}\right)$ are cross-intersecting and $|\mathcal{G}|>\left(\begin{array}{l}n-1 \\ k-1\end{array}\right)-\left(\begin{array}{l}n-k \\ k-1\end{array}\right)$. Then

$$
|\mathcal{H}| \leq k-1 \text {. }
$$

Proof. The first $\left(\begin{array}{l}n-1 \\ k-1\end{array}\right)-\left(\begin{array}{l}n-k \\ k-1\end{array}\right)=\left(\begin{array}{l}n-2 \\ k-2\end{array}\right)+\cdots+\left(\begin{array}{l}n-k \\ k-2\end{array}\right)$ subsets $G \in\left(\begin{array}{c}{[n-1]} \\ k-1\end{array}\right)$ are $\mathcal{L}\left(n-1, k-1,\left(\begin{array}{c}n-1 \\ k-1\end{array}\right)-\left(\begin{array}{c}n-k \\ k-1\end{array}\right)\right)=\left\{G \in\left(\begin{array}{c}n-1] \\ k-1\end{array}\right): G \cap[k-1] \neq \emptyset\right\}$. The next $(k-1)$-set is $[k, 2 k-2]$. The only $k$-subsets intersecting all of them are $[k-1] \cup\{j\}, k \leq j \leq 2 k-2$.

The proof of Theorem 1.4 (i) is based on the following result extending the Hilton-Milner Theorem to two families. 
Mörs Theorem $([\mathbf{M}])$. Suppose that $\mathcal{A}, \mathcal{B} \subset\left(\begin{array}{c}{[n]} \\ k\end{array}\right)$ are cross-intersecting, $n>2 k>0$ and neither $\mathcal{A}$ nor $\mathcal{B}$ is a star. Then

$$
\min \{|\mathcal{A}|,|\mathcal{B}|\} \leq\left(\begin{array}{l}
n-1 \\
k-1
\end{array}\right)-\left(\begin{array}{c}
n-k-1 \\
k-1
\end{array}\right)+1
$$

Let us note that for $k=1$ all the problems considered so far are trivial. For $k=2$, and neither $\mathcal{A}$ nor $\mathcal{B}$ being a star, either $\mathcal{A}=\mathcal{B}=\left(\begin{array}{l}T \\ 2\end{array}\right)$ for a 3-element set $T$, or one of $\mathcal{A}$ and $\mathcal{B}$ consists of two pairwise disjoint 2-sets and the other is a subset of the four 2-sets intersecting both. Therefore from now on we are going to assume $k \geq 3$.

In a recent work FK2 we extended the Mörs Theorem using the notion of diversity (cf. the definition and statement in Section 4). This result appears to be essential in establishing the exact value of $f^{*}(n, k):=\min \{|\mathcal{A}|,|\mathcal{B}|$ : $\mathcal{A}, \mathcal{B} \subset\left(\begin{array}{c}{[n]} \\ k\end{array}\right), \mathcal{A} \cap \mathcal{B}=\emptyset, \mathcal{A}$ and $\mathcal{B}$ are cross-intersecting, and neither of them is a star\}.

Proposition 2.2. For $n>2 k$ one has

$$
f^{*}(n, k) \geq\left\lfloor\frac{1}{2}\left(\left(\begin{array}{l}
n-1 \\
k-1
\end{array}\right)-\left(\begin{array}{c}
n-2 k \\
k-1
\end{array}\right)\right)\right\rfloor+1 .
$$

\section{The proof of Theorem 1.4 and Proposition 2.2}

Let $\mathcal{A}, \mathcal{B} \subset\left(\begin{array}{c}{[n]} \\ k\end{array}\right)$ be disjoint and cross-intersecting. If one of them (say $\mathcal{A}$ ) is a star then there are two cases. For definiteness suppose $1 \in A$ for all $A \in \mathcal{A}$. Should $1 \in B$ hold for all $B \in \mathcal{B}$ then $|\mathcal{A} \cup \mathcal{B}| \leq\left(\begin{array}{c}n-1 \\ k-1\end{array}\right)$ implies (1.5).

If there is some $B_{0} \in \mathcal{B}$ with $1 \notin B_{0}$ then the cross-intersecting property implies

$$
|\mathcal{A}| \leq\left(\begin{array}{l}
n-1 \\
k-1
\end{array}\right)-\left(\begin{array}{c}
n-k-1 \\
k-1
\end{array}\right) .
$$

On the other hand, if neither of $\mathcal{A}$ and $\mathcal{B}$ is a star, we may apply the Mörs Theorem and get the bound (2.2). Consequently, if

$$
\left(\begin{array}{l}
n-1 \\
k-1
\end{array}\right)-\left(\begin{array}{c}
n-k-1 \\
k-1
\end{array}\right)+1 \leq \frac{1}{2}\left(\begin{array}{l}
n-1 \\
k-1
\end{array}\right)
$$


then (1.5) holds. Equivalently

$$
\left(\begin{array}{c}
n-k-1 \\
k-1
\end{array}\right) \geq \frac{1}{2}\left(\begin{array}{l}
n-1 \\
k-1
\end{array}\right)+1
$$

Recall the inequality $1+x<e^{x}$ valid for all $x$. Suppose that $n \geq c\left(k^{2}-k\right)+$ $2 k-1$. Then

$$
\left(\begin{array}{c}
n-1 \\
k-1
\end{array}\right) /\left(\begin{array}{c}
n-k-1 \\
k-1
\end{array}\right)=\prod_{1 \leq i \leq k-1} 1+\frac{k}{n-k-i}<\left(1+\frac{k}{c k(k-1)}\right)^{k-1}<e^{\frac{1}{c}}=2 .
$$

This proves (3.2) for the corresonding range thereby establishing Theorem 1.4 (i).

Let us now prove that for $n \leq c(k-1)^{2}+1$,

$$
\left(\begin{array}{l}
n-1 \\
k-1
\end{array}\right)-\left(\begin{array}{l}
n-k \\
k-1
\end{array}\right)>\frac{1}{2}\left(\begin{array}{l}
n-1 \\
k-1
\end{array}\right) \text {. }
$$

Equivalently,

$$
\left(\begin{array}{l}
n-k \\
k-1
\end{array}\right)<\frac{1}{2}\left(\begin{array}{l}
n-1 \\
k-1
\end{array}\right)
$$

We derive (3.4) from the following chain of equalities and inequalities:

$$
\begin{gathered}
\frac{\left(\begin{array}{l}
n-k \\
k-1
\end{array}\right)}{\left(\begin{array}{l}
n-1 \\
k-1
\end{array}\right)}<\left(\frac{n-k}{n-1}\right)^{k-1}=\left(1-\frac{k-1}{n-1}\right)^{k-1} \leq\left(1-\frac{k-1}{c(k-1)^{2}}\right)^{k-1} \\
=\left(1-\frac{1}{c(k-1)}\right)^{k-1}<e^{-\frac{1}{c}}=\frac{1}{2}
\end{gathered}
$$

To use (3.3) let us define two families $\mathcal{F}$ and $\mathcal{G}$.

$$
\begin{aligned}
& \mathcal{F}=\left\{F \in\left(\begin{array}{c}
{[n]} \\
k
\end{array}\right): F \cap[k]=\{1\} \text { or }\{2,3, \ldots, k\}\right\}, \\
& \mathcal{G}=\left\{G \in\left(\begin{array}{c}
{[n]} \\
k
\end{array}\right): 1 \in G, G \cap\{2,3, \ldots, k\} \neq \emptyset\right\} .
\end{aligned}
$$


Then

$$
|\mathcal{F}|+|\mathcal{G}|=\left(\begin{array}{l}
n-1 \\
k-1
\end{array}\right)+n-k
$$

and the families $\mathcal{F}, \mathcal{G}$ are cross-intersecting while $\mathcal{G}$ is intersecting. In view of $(\underline{3.3}),|\mathcal{G}|>\frac{1}{2}\left(\begin{array}{c}n-1 \\ k-1\end{array}\right)$.

If $|\mathcal{F}|>\frac{1}{2}\left(\begin{array}{c}n-1 \\ k-1\end{array}\right)$ then we are done.

Suppose $|\mathcal{F}| \leq \frac{1}{2}\left(\begin{array}{l}n-1 \\ k-1\end{array}\right)$ and let $\mathcal{G}_{0} \subset \mathcal{G}$ be an arbitrary subfamily satisfying $\left|\mathcal{G}_{0}\right|=\left\lfloor\frac{1}{2}\left(\begin{array}{c}n-1 \\ k-1\end{array}\right)\right\rfloor+1-|\mathcal{F}|$. Set $\mathcal{A}=\mathcal{F} \cup \mathcal{G}_{0}, \mathcal{B}=\mathcal{G} \backslash \mathcal{G}_{0}$. By definition $|\mathcal{A}|=\left\lfloor\frac{1}{2}\left(\begin{array}{l}n-1 \\ k-1\end{array}\right)\right\rfloor+1$ and $(\underline{3.5})$ implies $|\mathcal{B}|>\frac{1}{2}\left(\begin{array}{l}n-1 \\ k-1\end{array}\right)$ as well. This completes the proof of Theorem 1.4 .

Let us turn to the proof of Proposition 2.2 .

Let $P, Q \in\left(\begin{array}{c}{[2 n]} \\ k\end{array}\right)$ satisfy $P \cap Q=\{2\}$. Define

$$
\begin{aligned}
& \mathcal{A}=\left\{A \in\left(\begin{array}{c}
{[n]} \\
k
\end{array}\right): 1 \in A, A \cap Q \neq \emptyset\right\} \cup\{P\}, \\
& \mathcal{B}=\left\{B \in\left(\begin{array}{c}
{[n]} \\
k
\end{array}\right): 1 \in B, B \cap P \neq \emptyset\right\} \cup\{Q\} .
\end{aligned}
$$

It is easy to see that $\mathcal{A}$ and $\mathcal{B}$ are cross-intersecting and neither of them is a star. However, they are not disjoint.

Noting $|\mathcal{A}|=|\mathcal{B}|$ and $|\mathcal{A} \cup \mathcal{B}|=\left(\begin{array}{c}n-1 \\ k-1\end{array}\right)-\left(\begin{array}{c}n-2 k \\ k-1\end{array}\right)+2$, one can remove equitably the members of $\mathcal{A} \cap \mathcal{B}$ from exactly one of the two families to obtain $\mathcal{A}_{0} \subset \mathcal{A}, \mathcal{B}_{0} \subset \mathcal{B}$,

$$
\begin{aligned}
& \left|\mathcal{A}_{0}\right|=\left\lfloor\frac{1}{2}\left(\left(\begin{array}{l}
n-1 \\
k-1
\end{array}\right)-\left(\begin{array}{c}
n-2 k \\
k-1
\end{array}\right)\right)\right\rfloor+1, \\
& \left|\mathcal{B}_{0}\right|=\left[\frac{1}{2}\left(\left(\begin{array}{l}
n-1 \\
k-1
\end{array}\right)-\left(\begin{array}{c}
n-2 k \\
k-1
\end{array}\right)\right)\right]+1 .
\end{aligned}
$$

Obviously, $\mathcal{A}_{0}$ and $\mathcal{B}_{0}$ are cross-intersecting and for $k \geq 2$ neither of them is a star. This concludes the proof of (2.3) .

\section{In the grey zone}

In the previous section we proved Theorem 1.4. To be more exact, we proved that

$$
f(n, k)=\left\lfloor\frac{1}{2}\left(\begin{array}{l}
n-1 \\
k-1
\end{array}\right)\right\rfloor \quad \text { if } \quad \frac{1}{2}\left(\begin{array}{l}
n-1 \\
k-1
\end{array}\right) \geq\left(\begin{array}{c}
n-1 \\
k-1
\end{array}\right)-\left(\begin{array}{c}
n-k-1 \\
k-1
\end{array}\right)
$$


and also

$$
f(n, k)>\frac{1}{2}\left(\begin{array}{l}
n-1 \\
k-1
\end{array}\right) \quad \text { if } \quad \frac{1}{2}\left(\begin{array}{l}
n-1 \\
k-1
\end{array}\right) \leq\left(\begin{array}{l}
n-1 \\
k-1
\end{array}\right)-\left(\begin{array}{l}
n-k \\
k-1
\end{array}\right) .
$$

Let us try and say something about the "grey zone", about the narrow range where

$$
\left(\begin{array}{l}
n-1 \\
k-1
\end{array}\right)-\left(\begin{array}{l}
n-k \\
k-1
\end{array}\right)<\frac{1}{2}\left(\begin{array}{l}
n-1 \\
k-1
\end{array}\right)<\left(\begin{array}{l}
n-1 \\
k-1
\end{array}\right)-\left(\begin{array}{c}
n-k-1 \\
k-1
\end{array}\right)
$$

or equivalently

$$
\left(\begin{array}{c}
n-k-1 \\
k-1
\end{array}\right)<\frac{1}{2}\left(\begin{array}{l}
n-1 \\
k-1
\end{array}\right)<\left(\begin{array}{l}
n-k \\
k-1
\end{array}\right) .
$$

Let us prove that even if (1.5) fails it is almost true.

Proposition 4.1. Suppose that (4.1) hold for the pair $(n, k), n>2 k$. Then

$$
f(n, k) \leq \frac{1}{2}\left(\left(\begin{array}{l}
n-1 \\
k-1
\end{array}\right)+n-k-1\right) .
$$

Proof. Let again $\mathcal{A}, \mathcal{B} \subset\left(\begin{array}{c}{[n]} \\ k\end{array}\right)$ be disjoint and cross-intersecting, moreover, $\min \{|\mathcal{A}|,|\mathcal{B}|\} \geq \frac{1}{2}\left(\begin{array}{c}n-1 \\ k-1\end{array}\right)$. If both are stars then (1.5) holds. Suppose now that $\mathcal{A}$ is a star where $n \in A$ for all $A \in \mathcal{A}$. In view of (4.1) we may apply Corollary 2.1 with $\mathcal{G}=\mathcal{A}(n)=\{A \backslash\{n\}: A \in \mathcal{A}\}, \mathcal{H}=\mathcal{B}(\bar{n})=\{B \in \mathcal{B}:$ $n \notin B\}$. This gives $|\mathcal{H}| \leq k-1$. Except for $\mathcal{H}$, all members of $\mathcal{A} \cup \mathcal{B}$ contain $n$. Thus we infer

$$
|\mathcal{A} \cup \mathcal{B}| \leq\left(\begin{array}{l}
n-1 \\
k-1
\end{array}\right)+k-1 .
$$

Since $\mathcal{A}$ and $\mathcal{B}$ are disjoint, (4.3) follows.

The case that remains is when neither $\mathcal{A}$ nor $\mathcal{B}$ is a star. To deal with this case we need the notion of diversity and the extension of Mörs Theorem.

Definition 4.2. For a family $\mathcal{F} \in 2^{[n]}$ define its diversity $\gamma(\mathcal{F})$ by $\gamma(\mathcal{F})=$ $\min \{|\mathcal{F}(\bar{i})|: i \in[n]\}$.

It should be clear that $\mathcal{F}$ is a star iff $\gamma(\mathcal{F})=0$. 
Theorem 4.3 ([FK2]). Suppose that $\mathcal{A}, \mathcal{B} \subset\left(\begin{array}{c}{[n]} \\ k\end{array}\right)$ are cross-intersecting, $n>2 k, k>3$. Let $u$ be an integer, $3 \leq u \leq k$ and suppose that

$$
\min \{|\mathcal{A}|,|\mathcal{B}|\} \geq\left(\begin{array}{l}
n-1 \\
k-1
\end{array}\right)-\left(\begin{array}{c}
n-u-1 \\
k-1
\end{array}\right)+\left(\begin{array}{l}
n-u-1 \\
n-k-1
\end{array}\right) .
$$

Then either equality holds in (4.4) for both $\mathcal{A}$ and $\mathcal{B}$ or

$$
\max \{\gamma(\mathcal{A}), \gamma(\mathcal{B})\}<\left(\begin{array}{l}
n-u-1 \\
n-k-1
\end{array}\right),
$$

moreover both families share the same (unique) element of maximum degree.

Let us return to the proof of Proposition 4.1. We apply Theorem 4.3 with $u=k-1$. If

$$
\min \{|\mathcal{A}|,|\mathcal{B}|\} \leq\left(\begin{array}{l}
n-1 \\
k-1
\end{array}\right)-\left(\begin{array}{l}
n-k \\
k-1
\end{array}\right)+\left(\begin{array}{c}
n-k \\
n-k-1
\end{array}\right)
$$

then via (4.1)

$$
\min \{|\mathcal{A}|,|\mathcal{B}|\} \leq \frac{1}{2}\left(\begin{array}{l}
n-1 \\
k-1
\end{array}\right)+n-k-1
$$

follows.

In the opposite case from (4.4) we derive

$$
\max \{\gamma(\mathcal{A}), \gamma(\mathcal{B})\} \leq n-k-1 .
$$

By symmetry we may assume

$$
\max \{|\mathcal{A}(\bar{n})|,|\mathcal{B}(\bar{n})|\} \leq n-k-1 .
$$

By disjointness of $\mathcal{A}$ and $\mathcal{B}$ we infer

$$
|\mathcal{A}(n)|+|\mathcal{B}(n)| \leq\left(\begin{array}{l}
n-1 \\
k-1
\end{array}\right) .
$$

Thus

$$
\frac{|\mathcal{A}|+|\mathcal{B}|}{2} \leq \frac{1}{2}\left(\begin{array}{l}
n-1 \\
k-1
\end{array}\right)+n-k-1
$$

follows. 


\section{Determining $f^{*}(n, k)$ for $n \geq k(k+5), k \geq 5$}

Throughout this section let $k \geq 5, n \geq k(k+5)$ and let $\mathcal{A}, \mathcal{B} \subset\left(\begin{array}{c}{[n]} \\ k\end{array}\right)$ be disjoint, cross-intersecting. Moreover we assume that neither of $\mathcal{A}$ and $\mathcal{B}$ is a star.

Since we are trying to determine $f^{*}(n, k)$, in view of Proposition 2.2 we may assume that

$$
\min \{|\mathcal{A}|,|\mathcal{B}|\}>\frac{1}{2}\left(\left(\begin{array}{c}
n-1 \\
k-1
\end{array}\right)-\left(\begin{array}{c}
n-2 k \\
k-1
\end{array}\right)\right)
$$

First we show that the above conditions on $n$ and $k$ guarantee that Theorem 4.3 can be applied with some $3 \leq u \leq k$.

\section{Claim 5.1.}

$$
\min \{|\mathcal{A}|,|\mathcal{B}|\}>\left(\begin{array}{l}
n-1 \\
k-1
\end{array}\right)-\left(\begin{array}{c}
n-4 \\
k-1
\end{array}\right)-\left(\begin{array}{c}
n-4 \\
n-k-1
\end{array}\right) .
$$

Proof. One can rewrite the RHS as

$$
\left(\begin{array}{l}
n-2 \\
k-2
\end{array}\right)+\left(\begin{array}{l}
n-3 \\
k-2
\end{array}\right)+\left(\begin{array}{l}
n-4 \\
k-2
\end{array}\right)+\left(\begin{array}{l}
n-4 \\
k-3
\end{array}\right)=\left(\begin{array}{l}
n-2 \\
k-2
\end{array}\right)+2\left(\begin{array}{l}
n-3 \\
k-2
\end{array}\right)<3\left(\begin{array}{l}
n-2 \\
k-2
\end{array}\right) .
$$

In view of (5.1) it is sufficient to show that

$$
\left(\begin{array}{l}
n-2 \\
k-2
\end{array}\right)+\left(\begin{array}{l}
n-3 \\
k-2
\end{array}\right)+\cdots+\left(\begin{array}{c}
n-10 \\
k-2
\end{array}\right)>6\left(\begin{array}{l}
n-2 \\
k-2
\end{array}\right)
$$

Noting that $\left(\begin{array}{c}n-i-1 \\ k-2\end{array}\right) /\left(\begin{array}{c}n-i \\ k-2\end{array}\right)=1-\frac{k-2}{n-i} \geq 1-\frac{k-2}{n-8}$ for $2 \leq i \leq 8$, using $n \geq k^{2}+5 k>(k-2)(k+7)+8$ we infer that the above ratio is always more than

$$
1-\frac{k-2}{(k-2)(k+7)}=1-\frac{1}{k+7} \geq \frac{11}{12} .
$$

Now (15.3) follows from $1+\frac{11}{12}+\left(\frac{11}{12}\right)^{2}+\cdots+\left(\frac{11}{12}\right)^{7}=12 \cdot\left(1-\left(\frac{11}{12}\right)^{8}\right)$ and $\left(\frac{11}{12}\right)^{8}<\frac{1}{2}$.

Now let $u$ be the maximal integer, $3 \leq u \leq k$ such that

$$
\min \{|\mathcal{A}|,|\mathcal{B}|\}>\left(\begin{array}{l}
n-1 \\
k-1
\end{array}\right)-\left(\begin{array}{c}
n-u-1 \\
k-1
\end{array}\right)+\left(\begin{array}{l}
n-u-1 \\
n-k-1
\end{array}\right) .
$$


In view of Theorem 4.3 we may assume that

$$
\max \{|\mathcal{A}(\overline{1})|,|\mathcal{B}(\overline{1})|\}<\left(\begin{array}{l}
n-u-1 \\
n-k-1
\end{array}\right) .
$$

Since neither $\mathcal{A}$ nor $\mathcal{B}$ is a star, $u<k$ follows.

Our next goal is to show that for $A \in \mathcal{A}(\overline{1})$ and $B \in \mathcal{B}(\overline{1})$ necessarily

$$
|A \cap B|=1 \text {. }
$$

Suppose for contradiction that $|A \cap B| \geq 2$. Set $D=A \cup B$. Note that $|D|=2 k-|A \cap B| \leq 2 k-2$.

The cross-intersecting property implies that every $C \in(\mathcal{A}(1) \cup \mathcal{B}(1))$ satisfies $D \cap C \neq \emptyset$. Consequently,

$$
|\mathcal{A}(1) \cup \mathcal{B}(1)| \leq\left(\begin{array}{c}
n-1 \\
k-1
\end{array}\right)-\left(\begin{array}{c}
n-2 k+1 \\
k-1
\end{array}\right)=\left(\begin{array}{c}
n-1 \\
k-1
\end{array}\right)-\left(\begin{array}{c}
n-2 k \\
k-1
\end{array}\right)-\left(\begin{array}{c}
n-2 k \\
k-2
\end{array}\right) .
$$

By (5.4) we have

$$
|\mathcal{A}(\overline{1})|+|\mathcal{B}(\overline{1})|<\left(\begin{array}{l}
n-4 \\
k-4
\end{array}\right) .
$$

Comparing with (5.1) we infer

$$
2\left(\begin{array}{c}
n-4 \\
k-4
\end{array}\right)>\left(\begin{array}{c}
n-2 k \\
k-2
\end{array}\right) .
$$

However, simple computation shows that this inequality fails for $n \geq k(k+5)$.

Indeed,

$$
\frac{\left(\begin{array}{c}
n-2 k \\
k-2
\end{array}\right)}{\left(\begin{array}{l}
n-4 \\
k-4
\end{array}\right)}=\frac{(n-2 k)(n-2 k-1)}{(k-2)(k-3)} \prod_{2 \leq i \leq k-3}\left(1-\frac{2(k-1)}{n-2-i}\right) .
$$

For $k \geq 5$ and $n \geq k(k+5), \frac{n-2 k-1}{k-3}>\frac{n-2 k}{k-2}>k+3 \geq 8$. Also $\frac{2(k-1)}{n-(k-1)}<\frac{2}{k+5}$ and $\left(1-\frac{2}{k+5}\right)^{k-2}>e^{-2}$. These show that

$$
\left(\begin{array}{c}
n-2 k \\
k-2
\end{array}\right)>\left(\begin{array}{l}
n-4 \\
k-4
\end{array}\right)\left(\frac{8}{e}\right)^{2},
$$

contradicting (5.7). Now (5.5) is proved. 
Claim 5.2. If $A, A^{\prime} \in \mathcal{A}(\overline{1})$ and $B, B^{\prime} \in \mathcal{B}(\overline{1})$ then

$$
A \cup B=A^{\prime} \cup B^{\prime} .
$$

Proof. Set $D=A \cup B, D^{\prime}=A^{\prime} \cup B^{\prime}$ and suppose indirectly $D \neq D^{\prime}$. As we pointed out before, the cross-intersecting property implies both $C \cap D \neq \emptyset$ and $C \cap D^{\prime} \neq \emptyset$ for all $C \in \mathcal{A}(1) \cup \mathcal{B}(1)$. It is easy to see that the total number of $(k-1)$-subsets $\tilde{C} \subset[2 n]$, intersecting both $D$ and $D^{\prime}$ is largest if $\left|D \cap D^{\prime}\right|=2 k-2$.

In that case the number is

$$
\begin{gathered}
\left(\begin{array}{l}
n-1 \\
k-1
\end{array}\right)-\left(\begin{array}{c}
n-2 k+1 \\
k-1
\end{array}\right)+\left(\begin{array}{c}
n-2 k-1 \\
k-3
\end{array}\right) \\
=\left(\begin{array}{c}
n-1 \\
k-1
\end{array}\right)-\left(\begin{array}{c}
n-2 k \\
k-1
\end{array}\right)-\left(\left(\begin{array}{c}
n-2 k \\
k-2
\end{array}\right)-\left(\begin{array}{c}
n-2 k-1 \\
k-3
\end{array}\right)\right) .
\end{gathered}
$$

It is only a slight difference with respect to (5.6) and we can get a contradiction in exactly the same way.

Claim 5.3. $\min \{|\mathcal{A}(\overline{1})|,|\mathcal{B}(\overline{1})|\}=1$.

Proof. Suppose the contrary. WLOG let $[2, k+1] \in \mathcal{A}(\overline{1}),[k+1,2 k] \in \mathcal{B}(\overline{1})$. Choose some different $A \in \mathcal{A}(\overline{1})$ and $B \in \mathcal{B}(\overline{1})$. By Claim [5.2, $A \cup B=[2,2 k]$. By (5.5), $A \cap B=\{j\}$ for some $j \in[2,2 k]$.

In the case $j=k+1, A \neq[2, k+1]$ implies $|A \cap[k+1,2 k]| \geq 2$, contradicting (5.5). Suppose by symmetry $2 \leq j \leq k$. Now (5.5) implies $[2, k+1] \cap B=\{j\}$ whence $B=\{j\} \cup[k+2,2 k]$. From $A \cap B=\{j\}, A=$ $[2, k+1]$ follows which is in contradiction with our choice $A \neq[2, k+1]$.

By symmetry, let us suppose that $|\mathcal{A}(\overline{1})|=1$. WLOG let $[2, k+1]$ be the unique member of $\mathcal{A}(\overline{1})$ and let $[k+1,2 k]$ be one of the members of $\mathcal{B}(\overline{1})$. Using Claim 5.2 and (5.5) we infer that

$$
\mathcal{B}(\overline{1}) \subset\{\{j\} \cup[k+2,2 k]: 2 \leq j \leq k+1\},
$$

in particular

$$
|\mathcal{B}(\overline{1})| \leq k
$$

Together with

$$
|\mathcal{A}(1) \cup \mathcal{B}(1)| \leq\left(\begin{array}{c}
n-1 \\
k-1
\end{array}\right)-\left(\begin{array}{c}
n-2 k \\
k-1
\end{array}\right)
$$


this implies

$$
\begin{aligned}
|\mathcal{A}|+|\mathcal{B}| & \leq\left(\begin{array}{l}
n-1 \\
k-1
\end{array}\right)-\left(\begin{array}{c}
n-2 k \\
k-1
\end{array}\right)+1+|\mathcal{B}(\overline{1})| \\
& \leq\left(\begin{array}{c}
n-1 \\
k-1
\end{array}\right)-\left(\begin{array}{c}
n-2 k \\
k-1
\end{array}\right)+1+k .
\end{aligned}
$$

In the case $|\mathcal{B}(\overline{1})|=1$ we infer

$$
\min \{|\mathcal{A}|,|\mathcal{B}|\} \leq\left\lfloor\frac{1}{2}\left(\left(\begin{array}{c}
n-1 \\
k-1
\end{array}\right)-\left(\begin{array}{c}
n-2 k \\
k-1
\end{array}\right)\right)\right\rfloor+1
$$

in accordance with (2.3) .

Let us show that for $n \geq k^{3}$ the RHS of the last displayed inequality is the value of $f^{*}(n, k)$.

Theorem 5.4. For $n \geq k^{3}$

$$
f^{*}(n, k)=\left\lfloor\frac{1}{2}\left(\left(\begin{array}{c}
n-1 \\
n-k
\end{array}\right)-\left(\begin{array}{c}
n-2 k \\
k-1
\end{array}\right)\right)\right\rfloor+1 .
$$

Proof. In view of (5.10), all we have to show is $|\mathcal{B}(\overline{1})|=1$. Suppose for contradiction $|\mathcal{B}(\overline{1})| \geq 2$. WLOG $[k+1,2 k]$ and $\{k\} \cup[k+2,2 k]$ belong to $|\mathcal{B}(\overline{1})|$. Then for $A \in \mathcal{A}(1)$ either

$$
\begin{array}{ll}
A \cap[k+2,2 k] \neq \emptyset & \text { or } \\
A \cap[k+2,2 k]=\emptyset & \text { but }\{k, k+1\} \subset A .
\end{array}
$$

The number of $(k-1)$-sets $A \subset[2, n]$ satisfying these conditions is $\left(\begin{array}{l}n-1 \\ k-1\end{array}\right)$ $\left(\begin{array}{c}n-k \\ k-1\end{array}\right)+\left(\begin{array}{c}n-k-2 \\ k-3\end{array}\right)$. In view of $|\mathcal{A}(\overline{1})|=1$, to conclude the proof it is sufficient to show

$$
\left(\begin{array}{l}
n-1 \\
k-1
\end{array}\right)-\left(\begin{array}{c}
n-k \\
k-1
\end{array}\right)+\left(\begin{array}{c}
n-k-2 \\
k-3
\end{array}\right)<\frac{1}{2}\left(\left(\begin{array}{c}
n-1 \\
k-1
\end{array}\right)-\left(\begin{array}{c}
n-2 k \\
k-1
\end{array}\right)\right)-\frac{1}{2} .
$$

Equivalently,

$$
\left(\begin{array}{l}
n-2 \\
k-2
\end{array}\right)+\cdots+\left(\begin{array}{l}
n-k \\
k-2
\end{array}\right)<
$$

$$
\left(\begin{array}{c}
n-k-1 \\
k-2
\end{array}\right)+\left(\begin{array}{c}
n-k-2 \\
k-2
\end{array}\right)+\cdots+\left(\begin{array}{c}
n-2 k \\
k-2
\end{array}\right)-\left(2\left(\begin{array}{c}
n-k-2 \\
k-3
\end{array}\right)+1\right) \text {. }
$$


The last term in brackets is of smaller order of magnitude. E.g., for $n-k>$ $4\left(k^{2}-4\right)$ it is smaller than $\frac{1}{2(k+2)}\left(\begin{array}{c}n-k-1 \\ k-2\end{array}\right)$.

We break up $\left(\begin{array}{c}n-k-1 \\ k-2\end{array}\right)$ into $k+1$ equal parts and use $\frac{1}{k+1}\left(\begin{array}{c}n-k-1 \\ k-2\end{array}\right)>\frac{1}{k+1}\left(\begin{array}{c}n-k-j \\ k-2\end{array}\right)$ for $j \geq 2$. One of these terms we use to compensate for the last term in (15.12). Consequently, instead of (5.12) it suffices to show that

$$
\left(\begin{array}{l}
n-2 \\
k-2
\end{array}\right)+\cdots+\left(\begin{array}{c}
n-k \\
k-2
\end{array}\right)<\frac{k+2}{k+1}\left(\begin{array}{c}
n-k-2 \\
k-2
\end{array}\right)+\cdots+\frac{k+2}{k+1}\left(\begin{array}{c}
n-2 k \\
k-2
\end{array}\right) .
$$

This inequality follows once we show

$$
\left(\begin{array}{c}
n-k-j \\
k-2
\end{array}\right) /\left(\begin{array}{l}
n-j \\
k-2
\end{array}\right)>\frac{k+1}{k+2} \quad \text { for } 2 \leq j \leq k .
$$

Let us expand the LHS of (5.13) and use the Bernoulli inequality

$$
\frac{\left(\begin{array}{c}
n-k-j \\
k-2
\end{array}\right)}{\left(\begin{array}{c}
n-j \\
k-2
\end{array}\right)}=\prod_{0 \leq i \leq k-3}\left(1-\frac{k}{n-j-i}\right)>\left(1-\frac{k}{n-2 k}\right)^{k-2}>1-\frac{k(k-2)}{n-2 k} .
$$

Now $n \geq k^{3}$ implies $n-2 k \geq k^{3}-2 k>(k+2) k(k-2)$, i.e., the RHS is at least $1-\frac{1}{k+2}=\frac{k+1}{k+2}$ completing the proof.

Let us mention that our argument was essentially sharp, that is for $n<$ $(1-\varepsilon) k^{3}$ the inequality (5.11) would fail completely. That is the difference of the two sides would be much more than $k$. Consequently, imitating the proof of Proposition 2.2 we can show the following.

Proposition 5.5. For any $\varepsilon>0$ and $k>k_{0}(\varepsilon)$ in the range $k^{2}+5 k<n<$ $(1-\varepsilon) k^{3}$ one has

$$
f^{*}(n, k)=\left\lfloor\frac{1}{2}\left(\left(\begin{array}{c}
n-1 \\
k-1
\end{array}\right)-\left(\begin{array}{c}
n-2 k \\
k-1
\end{array}\right)+k+1\right)\right\rfloor .
$$

\section{References}

[D] D. E. Daykin, Erdős-Ko-Rado from Kruskal-Katona, Journal of Combinatorial Theory A 17 (1974), 254-255. 
[DF] D. E. Daykin, P. Frankl, Extremal sets of subsets satisfying conditions induced by a graph, in: Graph theory and combinatorics (Cambridge, 1983), pp. 107-126, Academic Press, London, 1984.

[F] P. Frankl, A new short proof for the Kruskal-Katona Theorem, Discrete Mathematics 48 (1984), 327-329.

[F87] P. Frankl, Erdős-Ko-Rado Theorem with conditions on the maximal degree, Journal of Combinatorial Theory A 46 (1987), N2, 252-263.

[FF1] P. Frankl, Z. Füredi, A new short proof of the EKR theorem, Journal of Combinatorial Theory A 119 (2012), 1388-1390.

[FF2] P. Frankl, Z. Füredi, Non-trivial intersecting families, Journal of Combinatorial Theory A 41 (1986), 150-153.

[FK1] P. Frankl, A. Kupavskii, A size-sensitive inequality for cross-intersecting families, European Journal of Combinatorics 62 (2017), 263-271.

[FK2] P. Frankl, A. Kupavskii, Diversity, arXiv:1811.01111

[Hi] A. J. W. Hilton, unpublished manuscript.

[HM] A. J. W. Hilton, E. C. Milner, Some intersection theorems for systems of finite sets, Quart. J. Math. Oxford 18 (1967), 369-384.

[H] H. Huang, Two extremal problems on intersecting families, European Journal of Combinatorics 76 (2019), 1-9.

[HK] G. Hurlbert, V. Kamat, New injective proofs of the Erdős-Ko-Rado and Hilton-Milner theorems, Discrete Mathematics 341 (2018), N6, 1749-1754.

[Ka1] G. O. H. Katona, A theorem on finite sets, in: Theory of Graphs, Proceedings, Colloq. Tihany, Hungary, 1966, pp. 187-207, Akadémiai Kiadó, Budapest/Academic Press, New York, 1968.

[Ka2] G.O.H. Katona, A simple proof of the Erdős-Chao Ko-Rado theorem, Journal of Combinatorial Theory B 13 (1972), 183-184.

[K17] A. Kupavskii, Problem posed at Conference in Sanya, China, 2017.

[KZ] A. Kupavskii, D. Zakharov, Regular bipartite graphs and intersecting families, Journal of Combinatorial Theory A 155 (2018), 180-189.

[Kr] J. B. Kruskal, The number of simplices in a complex, in: Mathematical Optimization Techniques, pp. 251-278, Univ. of California Press, Berkeley, 1963.

[M] M. Mörs, A generalization of a theorem of Kruskal, Graphs and Combinatorics 1 (1985), N1, 167-183.

[Py] L. Pyber, A new generalization of the Erdős-Ko-Rado theorem, Journal of Combinatorial Theory A 43 (1986), 85-90. 